\title{
LA CIUDADANÍA COSMOPOLITA COMO ESTRATEGIA PEDAGÓGICA SEGÚN EL MODELO DE NACIONES UNIDAS ${ }^{1}$
}

\author{
Ernesto Fajardo Pascagaza ${ }^{2}$ \\ Hans Leonardo Florian Sánchez ${ }^{3}$
}

Resumen: La investigación titulada El modelo de las naciones unidas como estrategia pedagógica para el desarrollo de la ciudadanía cosmopolita, a través de la competencia comunicativa en el Gimnasio Campestre Reino Británico de Tenjo- Cundinamarca, tiene como objetivo principal reconocer en el modelo de naciones unidas el desarrollo de procesos de ciudadanía cosmopolita a través de la competencia comunicativa. Esta indagación es de tipo cualitativo apoyada desde la metodología hermenéutica y el método de análisis del discurso, debido a que el cuerpo de investigación se centra en actos discursivos. El problema de investigación es de naturaleza discursiva- interpretativa, razón por la cual los instrumentos de recolección de información permitieron la creación de un corpus textual amplio el cual fue analizado a partir de distintas variables que emergieron en la investigación. Seguidamente, los resultados de este corpus textual fueron triangulados como forma de validar los resultados finales para arrojar conclusiones en las que se demuestran que el modelo de naciones unidas es una estrategia pedagógica pertinente para el desarrollo de la ciudadanía cosmopolita y las competencias comunicativas.

Palabras clave: educación ciudadana, competencia comunicativa, ciudadanía cosmopolita, modelo de naciones unidas, estrategia pedagógica.

1 Artículo producto de la investigación realizada al interior del Grupo de Investigación ALETHEIA de la Universidad Santo Tomás, en torno a la relación entre educación, ética y política

2 Doctorando en Educación, Doctorando en Filosofía, Magíster en Filosofía y Magíster en Educación. Docente del Departamento de Humanidades y Formación Integral de la USTA. Integrante del Grupo de Investigación ALETHEIA. Investigador principal. Correo electrónico: ernestofajardo@usantotomas.edu.co

3 Magister en Educación Universidad Militar Nueva Granada. Coinvestigador. zafra0727@gmail.com 


\title{
THE COSMOPOLITE CITIZENSHIP AS A PEDAGOGICAL STRATEGY ACCORDING TO THE UNITED NATIONS \\ MODEL
}

\begin{abstract}
The research entitled The model of the united nations as a pedagogical strategy for the development of cosmopolitan citizenship, through communicative competence in the Campestre Gymnasium British Kingdom of Tenjo-Cundinamarca, has as main objective to recognize in the model of united nations the development of processes of cosmopolitan citizenship through communicative competence. This inquiry is of a qualitative type supported by hermeneutical methodology and the method of discourse analysis, because the research body focuses on discursive acts. The research problem is of a discursive-interpretative nature, which is why the instruments for gathering information allowed the creation of a broad textual corpus which was analyzed from different variables that emerged in the research. Next, the results of this textual corpus were triangulated as a way to validate the final results to show conclusions that show that the United Nations model is a pedagogical strategy relevant to the development of cosmopolitan citizenship and communicative competencies.
\end{abstract}

Keywords: citizenship education, communicative competence, Cosmopolitan citizenship, model of the united nations, pedagogical strategy.

\section{Introducción}

El problema del cual surge la presente investigación encuentra su origen en la formación para la ciudadanía que se brinda desde los colegios, en tanto que se reconoce a la escuela como la institución que por excelencia moldea a los sujetos en seres aptos para la convivencia adecuada en la sociedad. En este sentido se plantea la pregunta fundante: ¿Cómo el modelo de las naciones unidas desarrolla procesos de ciudadanía cosmopolita a través de la competencia comunicativa en el Gimnasio Campestre Reino Británico? De esta manera se establece el objetivo de la investigación: Reconocer en el modelo de las naciones unidas el desarrollo de procesos de ciudadanía cosmopolita a través de la competencia comunicativa para asumir el proyecto como una estrategia pedagógica en el Gimnasio Campestre Reino Británico.

Para el desarrollo de la presente investigación, se abordaron una serie de fuentes bibliográficas con las que se estructuró el marco teórico teniendo en cuenta las Perspectivas y apuestas para la formación en Ciudadanía cosmopolita, la argumentación sobre las Competencias comunicativas y su relación con la ciudadanía así como los Aspectos metodológicos referentes al tipo de investigación, la población y muestra, los hallazgos, las conclusiones y los resultados.

La investigación es de corte cualitativo, dado que lo que se pretende es analizar las posibilidades que tienen los modelos de naciones unidas en el desarrollo de la competencia ciudadana contextualizada en la institución educativa y entenderla como la capacidad de desarrollo de una ciudadanía cosmopolita por medio de la comunicación, por lo que se debe resaltar que la naturaleza misma del problema de investigación es discursivo, lo cual va a conllevar a que esta investigación se 
enmarque en un paradigma interpretativo, es decir, de asignación de sentido. El argumento más importante por el cual se considera esta investigación de corte cualitativo es la pretensión que se tiene de ahondar en el desarrollo de la teoría de la ciudadanía cosmopolita y la competencia comunicativa como competencia ciudadana. El método utilizado para esta investigación está basado en la hermenéutica, específicamente en el análisis del discurso, en tanto que se pretende la comprensión de la vida social o de ciertas dinámicas de una comunidad por medio del análisis del texto.

\section{Perspectivas y apuestas para la formación en Ciudadanía cosmopolita.}

De acuerdo con esta idea, en años recientes se han ido incorporando en los colegios como estrategia pedagógica para la formación en ciudadanía los modelos de naciones unidas MUN, en los cuales se representa por parte de los estudiantes, el desarrollo de sesiones en distintos comités según el modelo de la organización de naciones unidas - ONU-. La formación para la ciudadanía cosmopolita permite comprender que todo ser humano es potencialmente un ciudadano del mundo por el hecho mismo de habitar y compartir el planeta tierra (Meyer, 2009) y a esto apunta el modelo de naciones unidas.

Sin embargo, cabe preguntarse: ¿Realmente los modelos de naciones unidas forman para la ciudadanía? Y en caso de que lo hagan: ¿Cómo los modelos de naciones unidas forman para la ciudadanía? ¿Para qué tipo de ciudadanía? Dar respuesta a estas cuestiones es de primordial importancia debido a que no podemos negar que nos movemos en un mundo de interacción constante con el otro y es de esa manera que la ciudadanía se convierte en un elemento fundamental para el adecuado desarrollo de la sociedad y la garantía de la existencia comunitaria.

Para analizar la variable de ciudadanía en la historia en Colombia, se puede usar como crisol el concepto de Gómez Buendía (1999) conocido como el Almendrón, entendido como una proto-identidad colombiana de la cual se resalta como característica primordial, un gran desarrollo de la racionalidad privada por encima de la razón publica, es decir, una predominancia de los intereses particulares sobre el bienestar comunitario, situación que ha conllevado al daño del tejido social colombiano y la emergencia de múltiples fenómenos como los grupos guerrilleros, el narcotráfico, el paramilitarismo, la corrupción y crímenes que afectan diariamente a los ciudadanos colombianos.

Si bien esta característica se encuentra presente en nuestra sociedad, uno de los deberes fundamentales de la educación es la formación de un sujeto capaz de vivir adecuadamente en sociedad, razón por la cual el ministerio nacional de educación MEN ha desarrollado desde hace varios años una serie de publicaciones, guías y estándares de competencias ciudadanas que se deben desplegar en el proceso educativo de los estudiantes, para dar cumplimiento a la carta de naciones unidas y la constitución política colombiana y sus desarrollos posteriores en la ley 115 de 1994 o también conocida como ley general de educación en Colombia acerca de los aspectos relacionados con la formación para ejercer la ciudadanía. 
Como una nueva estrategia pedagógica que favorece la formación de ciudadanía en los estudiantes de bachillerato se han empezado a desarrollar modelos de naciones unidas en instituciones educativas tanto de carácter público como privado con la esperanza que estas cumplan con el objetivo de la formación en ciudadanía, sin embargo, ello se ha tomado como un hecho factico socialmente aceptado y promovido por distintos entes como secretarías de educación, rectores, docentes entre otros agentes educativos. Aunque nunca se ha comprobado como verdadero, solamente se presume que estos modelos iniciados por la universidad de Harvard colaboran en la formación para el ejercicio de la ciudadanía.

Es ante dicha situación que se ubica la pregunta central de esta investigación, a saber: ¿Es posible formar para la ciudadanía cosmopolita por medio de la competencia comunicativa a partir de los modelos de Naciones Unidas? De esta pregunta hay dos elementos a analizar, a saber, primero el tipo de ciudadanía que se pretende estudiar y segundo la competencia comunicativa a analizar la cual se fundamenta en planteamientos de la filosofía del lenguaje y por lo tanto se distancia de las competencias ciudadanas programadas por el MEN.

Por esa misma razón, en este escrito se presentan los lineamientos del MEN acerca de las competencias ciudadanas así como los planteamientos de Guillermo Hoyos (2007) acerca de la formación en ciudadanía. Igualmente se analiza si estos modelos de naciones unidas son acordes a dicha propuesta. Por último se hace un acercamiento comprensivo a la competencia comunicativa como un camino hacia la ciudadanía y más particularmente a la ciudadanía cosmopolita desde las luces que brinda Cortina (1997). No resta decir que una de las principales defensoras del cosmopolitalismo es Nussbaum (1997) quien asume que hay habilidades fundamentales para ejercer una ciudadanía democrática. Ante todo, prima el pensamiento crítico como capacidad para razonar con lógica en el establecimiento consistente de razonamientos. Otra habilidad es la que permite comprender las diferencias que se establecen en las relaciones sociales así como las necesidades e intereses del género humano. En este sentido, se adquiere la habilidad para ponerse en el lugar del otro y comprender su historia de manera inteligente. (Nussbaum, 2006).

Inicialmente encontramos los dos documentos sobre competencias ciudadanas que han regido la formación ciudadana en Colombia, el primero de ellos Estándares Básicos de Competencias Ciudadanas: Formar para la ciudadanía iSi es posible! (MEN, 2004) el cual pretende brindar herramientas a los estudiantes para ser sujetos aptos en el momento de ejercer sus derechos y deberes en la vida cotidiana. Para desarrollar las competencias ciudadanas es necesario generar por medio de la educación dos elementos, a saber, conocimientos (Saber) y habilidades (saber hacer), ello con el fin de llegar a que los individuos generen una actuación constructiva para la sociedad democrática. Sin embargo, para obtener este tipo de actuación no basta la información, el conocimiento o el saber, sino que se debe complementar con el saber hacer, es decir, con habilidades de 4 tipos. Primera habilidad cognitiva, es decir, los procesos mentales; habilidad emocional, por la cual se identifican las emociones y se da respuesta a las mismas, habilidad comunicativa, 
por medio de la cual se posibilita el establecimiento de dialogo constructivo para la resolución de problemas y finalmente, la competencia integradora, que utiliza los elementos anteriores (información, procesos mentales, emociones y comunicación) para hallar la forma adecuada de dar manejo a los conflictos interpersonales.

El conocimiento y las habilidades para la resolución de conflictos se clasifican en tres tipos de competencias ciudadanas: convivencia y paz; participación y responsabilidad democrática; pluralidad, identidad y valoración de diferencias. Por medio de ellas se pretende que los estudiantes desarrollen consideración por los demás sujetos, que sean capaces de tomar decisiones, que respeten los derechos y normas vigentes de la sociedad y se relacionen con el resto de la sociedad desde el reconocimiento a la diversidad y el empleo de las mediaciones como la instancia cultural desde la cual se producen, crean y recrean significados y sentidos. (Barbero, 1991).

Los Estándares Básicos de Competencias Ciudadanas: Formar para la ciudadania iSi es posible! (MEN, 2004) se dan dentro del marco de la revolución educativa, la cual plantea la educación como camino para la paz. Por lo tanto, es necesario tener en cuenta que estos planteamientos se dan dentro del modelo educativo basado en competencias.

"Este modelo educativo considera que la realidad a conocer es parte de una gama de relaciones más complejas, la cual no es posible definir a partir de materias o asignaturas aisladas, sino mediante objetos de estudio, que han de entenderse como partes de un espacio problematizado y socialmente definido." (Moncada, 2010, p. 52)

En este sentido, los objetos de estudio en el modelo de competencias ciudadanas son problemas que pasan más allá de lo teórico hacía lo vivencial, es decir, el estudiante se ve interpelado por la realidad social que lo afecta y con la cual debe comprometerse para mejorarla y asumir que una de las principales características del modelo es la flexibilidad curricular en tanto que se desea trabajar problemas de manera interdisciplinar y por lo tanto, las habilidades no se desarrollan solamente desde una asignatura. Este planteamiento desde el MEN no se ha aplicado de manera adecuada, dado que la constitución y subsecuentemente el ministerio plantea la responsabilidad del desarrollo de las competencias ciudadanas en las asignaturas de constitución política, democracia, y ética.

El modelo basado en competencias trae consigo unos tipos de competencias que se deben trabajar en los estudiantes, las cuales son: Aprender a aprender, aprender a hacer, aprender a convivir y aprender a ser. Para Moncada, (2010): "Las competencias se basan en el interés que los estudiantes manifiesta por aprender, lo cual los lleva a interesarse por los problemas sociales y culturales y por contribuir con soluciones a los mismos.” (p. 56)

Aquí encontramos otra diferencia con lo planteado por el MEN en la que se habla del desarrollo de competencias ciudadanas desde dos ámbitos, a saber, primero los conocimientos y segundo las habilidades comunicativas, emocionales, cognitivas e integradoras y por lo tanto, se ve solamente el desarrollo de las habilidades de aprender a aprender (conocimientos) y aprender a hacer (habilidades). Hay un trasfondo del discurso de la modernidad, al estilo Kantiano en el que se plantea que 
la luz natural de la razón guiará el obrar humano hacia una acción coordinada con el deber ser planteado por la teoría conocida.

De igual manera, el programa de competencias ciudadanas - PCC- plantea que el conocimiento de los diferentes marcos organizativos, políticos, legales del Estado y el desarrollo de ciertas habilidades mencionadas con anterioridad, conllevarán al desarrollo de un ciudadano como es el deseado por la Constitución Política de 1991 y el MEN al sugerir que serán ciudadanos con valores éticos, que respeten lo público, ejerzan derechos al igual que cumplan responsabilidades, convivan en paz valorando la diferencia y siendo ciudadanos políticos participativos. (MEN. 2011).

En este sentido, nos encontramos con un modelo de ciudadano que ha sido planteado desde la constitución cumpliendo con el deber internacional adquirido con las Naciones Unidas al firmar la declaración de los Derechos Humanos, razón por la cual algunos de los fundamentos de la carta magna son: la dignidad humana y la democracia como formas de gobierno donde el poder es ostentado por los ciudadanos como pueblo soberano. En este sentido, la ciudadanía informal de la cual habla Jiménez (2003), logra avances en la misma concepción del ciudadano y de la reclamación de derechos. Una reclamación de derechos para la creación de ambientes de paz, una paz como punto de llegada por medio de las prácticas democráticas.

Las bases para las prácticas democráticas que plantea el MEN se encuentran en las Orientaciones para la institucionalización de las competencias ciudadanas Cartilla 1 en cinco diferentes ámbitos de los establecimientos educativos -EE- los cuales son la gestión institucional, las instancias de participación, el aula de clase, proyectos transversales y tiempo libre.

Hoyos (2007), realiza un análisis desde el paradigma cientificista determinando que las competencias ciudadanas se ponen al mismo nivel que las técnicas, las científicas y las lingüísticas y por lo tanto propone analizarlas desde otra perspectiva o paradigma educativo como el discursivo que se basa en la ética del discurso planteado por Habermas.

Desde este paradigma discursivo se pretende superar los paradigmas cientificistas y humanistas en la educación, dado que desde esta tercera vía se puede dar una solución diferente al problema de la deshumanización del hombre y la crisis moral. Para ello, se fundamenta la propuesta desde la moral de máximos y la ética de mínimos que desarrolla Cortina basada en la ética discursiva de Habermas. Así pues, desde la acción discursiva se puede comprender las estructuras comunicativas del mundo de la vida para facilitar la compresión de la sensibilidad moral y la tolerancia, reconociendo la diferencia y las diversas posibilidades de argumentación.

Es decir, desde el paradigma discursivo se pueden clasificar y comprender los contextos en los que se constituye la ciudadanía, dado que por medio de la comunicación se articulan o entrelazan los mundos de vida, permitiendo la creación de bases para el poder de la solidaridad ciudadana que desembocará en la interacción entre sociedad y Estado y de esta manera se optimice la participación 
ciudadana. Por lo tanto, en el planteamiento de Hoyos (2007), se resta fuerza al discurso de las competencias como elemento de exclusión o diferenciación. Para este autor, un modelo adecuado para la formación ciudadana debe surgir desde la inclusión, dado que al desear formar para la ciudadanía se debe reducir la brecha con respecto a los marginados o excluidos y permitirles también a ellos participar en el proceso de reconstruir el tejido social, en tanto que es la reconstrucción de lo público, entendiendo como público la red de intereses comunes que tienen individuos, organizaciones y Estado.

En la propuesta de Hoyos (2007), el elemento fundamental de la educación en ciudadanía es la competencia comunicativa, particularmente el dialogo desde donde logra articular las competencias cognitivas y emocionales, en cuanto que las emociones como el resentimiento, la indignación y la culpa se puedan comunicar y por medio de ellas se puede explicitar el sentido moral que debe ser justificado intersubjetivamente al tiempo que la comunicación argumentada puede generar acuerdos y consensos, es decir, conocimiento. Este conocimiento se obtiene a partir de procesos de comunicación intersubjetiva que permiten conocer lo correcto, lo veraz y lo objetivo.

Hoyos (2007), propone un paradigma discursivo de la educación basado en la propuesta de Cortina, es decir, en una moral de máximos y una ética de mínimos. Se debe tener en cuenta que para el desarrollo de esta propuesta es necesaria la competencia, pero una diferente a la propuesta desde el paradigma cientificista y el modelo educativo. Es aquí donde Hoyos habla sobre la competencia lingüística o comunicativa planteada por Chomsky y Hymes.

La competencia comunicativa se puede hallar en los modelos de naciones unidas, que son simulaciones del funcionamiento del sistema de las naciones unidas que se desarrollan por parte de estudiantes de bachillerato o universitarios, en los cuales los estudiantes asumen el rol de delegados de diferentes países ante esta organización internacional generando un foro de discusión en el cual los participantes se involucran en debates en torno a problemas que están siendo tratados por la ONU o que afectan a gran cantidad de personas y que merecen ser analizados para generar propuestas de solución, las cuales se convertirán en resoluciones y recomendaciones finales para los países.

El desarrollo mismo del modelo de naciones unidas pone en juego varios elementos que se han presentado hasta el momento, particularmente la posibilidad de que se construya ciudadanía cosmopolita, por medio de la competencia comunicativa-ciudadana. Dado que los estudiantes se han preparado investigando sobre los distintos países que le fueron asignados y han tomado una posición sobre las problemáticas mismas de sus naciones, se puede rastrear tres elementos que son características fundantes de la ciudadanía cosmopolita, a saber: el afecto por el género humano, contemplación de un sistema global de derechos y la construcción y/o defensa de instituciones supranacionales que defienden los derechos del ciudadano, pero a su vez los juzgan en el incumplimiento de sus deberes. 
Con esto se desea clarificar que los modelos de naciones unidas pueden ser una estrategia pedagógica que responde a las necesidades educativas contemporáneas, en tanto que los límites territoriales se han visto deteriorados, los gobiernos nacionales debilitados y los habitantes de un territorio han debilitado sus lazos locales ampliando así sus fronteras, lo cual ha permitido la emergencia de este nuevo modelo de ciudadanía que tiene incidencias fundamentales en la práctica de la participación cívica y las consecuentes relaciones que se establecen entre el ciudadano y el Estado a partir del sentido de pertenencia con los valores humanistas desde los marcos normativos internacionales. (Lagos, 2003). Se trata de aprender a vivir juntos para comprender la historia de los demás a partir de proyectos comunes respetando las tradiciones y valores de los demás. (Delors, 1996).

\section{Competencia y ciudadanía desde la perspectiva comunicativa.}

La competencia comunicativa surgió en el año 1965 cuando Noam Chomsky la definió como las capacidades y disposiciones para interpretar y actuar. Sobre este mismo concepto Hymes (1999), plantea que se desarrolla de acuerdo a las demandas del entorno. Igualmente plantea una crítica al modelo de la competencia comunicativa desde la teoría lingüística al referenciarla como una relación homogénea entre hablante y oyente, en tanto que manejan un mismo lenguaje y no se presentan errores gramaticales. De esta forma se puede evidenciar una predilección por la estructura (forma) dejando en un segundo plano la actuación lingüística, categorizada como un simple residuo.

De esta forma, Hymes (1999), pretende organizar de una nueva forma la relación entre la competencia lingüística, que plantea al sujeto como un ser abstracto aislado que se asienta en la forma gramatical y la actuación lingüística, considerada como el residuo llevado a cabo en el mundo social. Plantea la necesidad de una competencia diferencial para una comunidad lingüística heterogénea en tanto que depende de factores sociales, específicamente: la experiencia social, las necesidades y las motivaciones.

Por lo tanto, según este autor la competencia comunicativa se debe entender como la capacidad subyacente de una persona para interactuar con los otros. Esta competencia depende del conocimiento tácito y la motivación o la volición de los sujetos. Es de esta forma que en la propuesta de Hymes se encuentra una clara relación entre el conocimiento con la afectividad que se manifiesta en el uso mismo del lenguaje. En palabras del autor: "La competencia depende del conocimiento (tácito) y del uso (habilidad para éste). El conocimiento es por consiguiente diferente de la competencia (ya que es parte de ésta) y de la posibilidad sistemática (cuya relación es un asunto empírico)." (Hymes, 1999, p. 27).

Es decir, la competencia comunicativa trae consigo todo un entramado de habilidades, saberes, capacidades para participar en las relaciones interpersonales y en el entramado social, es decir, esta competencia permite establecer relaciones saludables y productivas. "La competencia comunicativa implica, entonces, una serie de procesos, saberes y experiencias de diversos tipos el que emisor-receptor deberá poner en juego 
para producir o comprender discursos adecuados a la situación y al contexto de comunicación" (Bermudezy González, 2011 p. 4)

La competencia comunicativa según Bermúdez y Gonzales (2011) se divide en dos dimensiones a saber, lingüística y estratégica. La dimensión lingüística es la referida a la gramática tradicional: morfología, sintaxis, fonética, fonología y semántica y a ello van aunados los elementos del contexto sociocultural, que influyen en la comprensión de los símbolos y significados. Por otra parte, la dimensión estratégica está relacionada con el uso efectivo de las formas de comunicación.

$\mathrm{Al}$ referir al uso efectivo de las formas de comunicación, se debe recordar los cuatro criterios planteados por Hymes (1999), a saber: primero, la posibilidad de un juicio dentro de un sistema gramatical, cultural y de acuerdo a la ocasión comunicativa. Segundo, la factibilidad del juicio en relación a la conducta cultural. Tercero, la pertinencia en tanto que corresponde a la necesidad de la situación comunicativa. Finalmente, la realización del acto comunicativo, en tanto que se puede cumplir con los tres criterios anteriores, pero aun así no realizar el acto comunicativo.

Cuando se hace referencia a una competencia comunicativa que es distinta al modelo cientificista adoptado desde el proyecto tunning para educación, la apuesta debe ser también radical en cuanto a un modelo distinto de ciudadanía, debido al deber que se tiene como educador de responder a las necesidades o problemas que se hallan en el entorno.

Es por ello que al plantear la formación en ciudadanía desde un tipo distinto de competencia se hace necesario reflexionar sobre el tipo de ciudadanía para la cual se pretende formar y por lo tanto el modelo de ciudadanía cosmopolita planteado por Cortina o cosmopolitismo cívico, que plantea un sistema global de derechos y deberes de carácter universal se convierte en el modelo referencial contextual particular pero también abierto a la ciudadanía universal. Por lo tanto, se hace necesario un sistema global de derechos y deberes acentuando en la necesidad de aceptar un pluralismo de cosmovisiones, lo cual puede ser factible si tenemos en cuenta que Benéitez (2010), plantea que "el afecto cosmopolita hacia el género bumano constituye nuestra lealtad primaria". Así para el ciudadano cosmopolita su lugar de nacimiento o donde habita son factores accidentales, en tanto que ejerce sus derechos bajo un sistema global. Sin embargo, el problema que se debe tener en cuenta es la construcción de instituciones globales.

La importancia de plantear un nuevo modelo de ciudadanía surge desde el proceso de globalización por el cual atraviesa la humanidad, dado que este proceso ha debilitado los lazos con el territorio y por lo tanto con el Estado. Esta realidad se observa en el creciente número de migraciones o en el marcado multiculturalismo contemporáneo que ha conllevado a que los derechos no se limiten al ámbito del Estado, quien anteriormente era garante de los mismos. Formarse para la ciudadanía del mundo permite comprender que el escenario global da cuenta de una compleja relación de interacciones sociales de todo orden, desde aquellas que generan ejercicios aproximativos de sociabilidad así como aquellos que crean realidades de 
inequidad e injusticia social a escala local y global. (Appadurai, 2000). Este modelo ofrece variables para percibir la ciudadanía global dentro de las variables y complejas identidades y compromisos desde lo local y global. (De Paz, 2007). Ahora bien, se trata igualmente de una preocupación por una ética de lo global (Sen, 2006), para la comprensión entre unos y otros (Delanty, 2006), para la sensibilidad intercultural y la adquisición de competencias interculturales dirigidas a los encuentros dialógicos protagónicos. (Bennett, 2004).

Si la concepción moderna de ciudadanía implicaba hacer parte de un Estado, una concepción contemporánea de la ciudadanía implicará la pertenencia a instituciones supranacionales, las cuales habrá que crear y asegurar mediante acuerdos, pero lo más importante es la educación en el cosmopolitismo. En este sentido: "la perspectiva cosmopolita es algo activo y reflexivo, en contraste con el cosmopolitismo banal que se despliega bajo la fachada de persistentes espacios, jurisdicciones y etiquetas nacionales" (Beck, 2006, 7). Es un modelo que busca defender un sistema global de derechos y deberes universales a partir de la aceptación pluralista y la tolerancia en las cosmovisiones. (Escobar, 2007).

Con base en esto, el modelo de ciudadanía cosmopolita es un referente adecuado para el desarrollo del presente trabajo investigativo, en tanto que los modelos de naciones unidas son la réplica a menor escala de una de estas instituciones supranacionales que permitan el desarrollo de una ciudadanía universal, la cual se basa en el actuar de acuerdo a los fines que cualquier humano podría querer. Con este modelo se pretende una interacción equitativa de acercamiento a las culturas compartiendo sus idearios diálogicos y de respeto mutuo, (UNESCO, 2006), dado que hay una creciente conciencia de interconexión natural del mundo que se habita (O’Byrne, 2003), y de las megatendencias cívicas (Kennedy, 1997), así como de preocupación y mente abierta ante las realidades apremiantes del mundo globalizado que exigen justicia, equidad y derechos humanos de manera incluyente. (Carvalho da Silva, 2008). Si bien, se entiende que este modelo es un trabajo en construcción, que como ya se ha dicho, estas instituciones por el momento están en proceso de creación o su trabajo aún es incipiente, se debe tener en cuenta que son una realidad palpable sobre el futuro de la humanidad y ayuda a comprender de una forma adecuada el momento actual en el que se encuentra la ciudadanía y sus prácticas.

Sin embargo, es necesario reconocer la gran influencia que ha jugado el proceso de globalización en la transformación de la práctica de la ciudadanía, como lo plantea Canclini (1991). Se han generado nuevas relaciones entre ciudadanía y consumo, por ejemplo, los ciudadanos que se identificaban previamente con el Estado y su cultura particular, han encontrado nuevas identidades en productos comerciales, lo cual ha debilitado aún más la relación ciudadano - Estado y ha generado un debilitamiento del Estado- Nación. Es de esta forma que el comercio ha logrado someter todos aquellos valores públicos que se consideran valiosos a su dinámica. En este sentido, el ciudadano se fundamenta actualmente en su rol económico en tanto que es capaz de producir y distribuir la cultura como un bien o un servicio cualquiera del mercado. 
Así pues, el rol de la ciudadanía se ha llevado hacia el consumo de la cultura, es decir, hacia la compra de identidad representada en los productos del mercado que permiten compartir sentido, los cuales se convierten en deseos y por lo tanto demanda de un consumidor al mercado. Es aquí donde se puede analizar que la ciudadanía pierde su centro en lo histórico- territorial y se juega ahora en la cultura de elites y la comunicación de masas.

Teniendo en cuenta que los cambios generados por el fenómeno de la globalización el cual ha desterritorializado la ciudadanía, fenómeno que ha permitido el surgimiento y fortalecimiento de este modelo cosmopolita, se hace necesario analizar o plantear una mejor manera de educar, de guiar y brindar a los estudiantes las herramientas, competencias o capacidades necesarias para vivir en la sociedad contemporánea, en la cual pueden ejercer derechos, pero deben cumplir con sus obligaciones.

Educar para el modelo de la ciudadanía cosmopolita es necesario (Appiah, 2008), porque permite que se abran espacios para el pensamiento crítico consciente, la imaginación de escenarios abiertos al diálogo y al debate (Walker, 2006), así como escenarios para reflexionar sobre los desafíos que conlleva vivir en una sociedad global interconectada y no reducida a comunidades políticas territorializadas por fronteras (UNESCO, 2014 ), que exigen nuevas formar de asumir y pensar la realidad desde todos las instancias posibles como las políticas, económicas, religiosas, culturales entre otras. (UNESCO, 2006).

\section{Aspectos metodológicos, hallazgos y resultados.}

El universo poblacional de esta investigación fueron todos los estudiantes de bachillerato del GCRB distribuidos en 20 debates estudiantiles., sin embargo, la muestra fue representativa, para la cual se tomaron 3 debates en particular, los cuales son los consejos de seguridad de los 3 distintos niveles en los que se desarrollará el modelo de naciones unidas. En estos niveles se cuentan con 65 estudiantes distribuidos de la siguiente manera: en nivel sexto y séptimo con 17 estudiantes, octavo y noveno con 24 estudiantes y décimo y undécimo con 24 estudiantes. Se toman los 3 consejos de seguridad dado que ellos comparten como temática común el conflicto armado en Siria y el terrorismo fundamentalista del Estado Islámico, temas los cuales ponen en juego el sistema político democrático de gran cantidad de naciones en la actualidad.

Estas mesas son las que mayor representación dan al tema de la ciudadanía en tanto que la condición de ciudadano solo en posible en sistemas democráticos y por esta razón se considera que en estos tres consejos de seguridad se evidenciarán claramente los elementos que se esperan analizar.

La investigación se plantea de corte cualitativo debido a que describe, analiza e interpreta los hechos ocurridos dentro del modelo de naciones unidas de la institución, evento que se caracteriza por los actos comunicativos de los estudiantes, es decir, se pretende analizar las posibilidades que tienen los modelos de naciones unidas en el desarrollo de la competencia ciudadana, entendida como la capacidad 
de desarrollo de una ciudadanía cosmopolita por medio de la comunicación, por lo que se debe resaltar que la naturaleza misma de problema de investigación es discursiva, lo cual conlleva a que esta investigación se enmarque en un paradigma interpretativo, es decir, de asignación de sentido.

Entendiendo esta característica investigativa, se adopta la metodología hermenéutica, específicamente el método de análisis crítico del discurso. Desde este método de investigación se deben analizar los textos en tres niveles, a saber: Sintáctico, el cual se encuentra relacionado con la forma propia del lenguaje y los niveles semántico y pragmático. Estos niveles se relacionan principalmente con el contenido y uso de la comunicación. El resultado de este análisis de tres niveles es la producción de un meta texto, en el cual se ha transformado el texto original.

Desde la postura de Van Dick (2003) el análisis crítico del discurso -ACDse entiende no solamente como un método, sino a su vez como una disciplina transversal, la cual se encarga de dar cuenta de las relaciones existentes entre los discursos y las estructuras sociales, ello ha permitido que su uso central se haya dado históricamente en el hecho de reivindicar la posición de los oprimidos. Sin embargo, como ya se manifestaba con anterioridad, el ACD es el método escogido para la presente investigación debido a que permite teorizar las relaciones del texto y el contexto, entendiendo por texto todos los acontecimientos comunicativos.

Para la creación del corpus textual se utilizaron tres instrumentos, a saber: papeles de posición, debates y grupo focal. Los papeles de posición y discursos de apertura son documentos escritos por los estudiantes en los cuales plantean y desarrollan el punto de vista del país del cual son delegados. Estos fueron recolectados, organizados de acuerdo con los tres niveles en los cuales se realizaron los debates. Además, se realizó la grabación de los debates de los tres niveles que fueron transcritos para realizar el análisis del mismo. Por otra parte, se realizó un grupo focal basado en una entrevista semiestructurada la cual fue validada por distintos expertos en educación.

Este corpus textual fue analizado de acuerdo con los planteamientos de distintos autores sobre el análisis crítico del discurso, el cual no cuenta con una estructura o procedimiento estándar, pero autores como Van Dick (2003) plantean algunas recomendaciones y explicaciones sobre el proceso de análisis del discurso. En este estudio se realizó el análisis semántico y pragmático de los discursos, principalmente, porque en las categorías de la investigación se plantea un análisis del uso efectivo de la comunicación, lo cual está directamente relacionado con el significado de lo que comunican los estudiantes y la intención con la que lo realizan. Cabe aclarar que para esta investigación se tomó una muestra representativa del total de 20 comités para debatir, se optó por analizar los 3 consejos de seguridad debido a la importancia contemporánea del tema de la seguridad internacional respecto a la amenaza de la guerra en siria y el estado islámico.

Posteriormente, se realizó en análisis de cada uno de los instrumentos arrojando resultados por categoría y triangulando los tres instrumentos. Es necesario tener en cuenta que se utilizaron dos super categorías, a saber: Competencia 
comunicativa, la cual se encuentra compuesta por las categorías de consensos, comunicación de emociones y uso efectivo de la comunicación, la cual a su vez se encuentra subdividido en las subcategorías de claridad y propósito comunicativo. La segunda super categoría es ciudadanía cosmopolita que se compone de las categorías sistema global de derechos e instituciones supranacionales.

El corpus textual creado fue analizado en distintas etapas. En primer momento, se realizó el análisis de los documentos escritos por los estudiantes (discursos de apertura y papeles de posición) de acuerdo con los tres niveles en los que se habían organizado a los estudiantes. En segundo momento, se realizó el estudio a las transcripciones de los tres debates del consejo de seguridad debido a que estos fueron seleccionados como muestra representativa. En tercer lugar, se desarrolló el estudio del grupo focal con la participación de algunos estudiantes de los distintos comités, para así finalmente realizar la triangulación de estos distintos momentos de análisis y poder comprender el comportamiento de las categorías de acuerdo a los tres niveles, por medio de estos tres instrumentos de recolección de información.

En relación con los documentos escritos por los estudiantes, se analizó el desarrollo de cada una de las categorías en los tres niveles. En este paso se evidenció que con el avance de los grados se nota mayor claridad respecto a la producción de discursos apropiados de acuerdo con el contexto, ello se debe a elementos tales como el conocimiento de los estudiantes sobre la posición de su país. Además en los documentos, se encuentran las propuestas de los estudiantes en las cuales ronda la idea del trabajo cooperativo internacional para dar solución a los problemas tratados. Sin embargo, estas ideas se plantean debido a la preocupación por los distintos conflictos que se viven en medio oriente y el terrorismo generado por este, particularmente por la forma en la que los conflictos y el terrorismo afectan a los pobladores y los convierten en víctimas.

Otro elemento importante a resaltar en los documentos escritos por los estudiantes es la actitud abierta al encuentro con las ideas de los demás por medio del diálogo y la negociación para generar acuerdos que detengan las violaciones de los derechos humanos y las afectaciones que en general las vive la población. Frente a estos planteamientos, los delegados manifiestan preocupación en dos sentidos, por un lado, la vida de los habitantes de las zonas en conflicto y por otra parte, los refugiados. Finalmente se evidencia que entre los estudiantes se encuentra la percepción que la cooperación internacional es la que permite dar solución adecuada y eficaz a los problemas.

En el segundo momento se realizó el análisis de las transcripciones de los debates de los tres consejos de seguridad; nuevamente este estudio fue realizado comparando el desarrollo de cada una de las categorías en los tres niveles de los debates. Entre los hallazgos cabe resaltar que varios estudiantes que no tenían claridad sobre el tema no lograron una preparación fructífera, lo cual se evidencia en el momento de participar en el debate, con intervenciones fuera de contexto y poca participación en la discusión. 
Por otra parte, respecto al objetivo del comité se encuentra que todas las mesas concuerdan en proteger los derechos humanos, dar solución a los conflictos y mantener la paz. Desde esta visión, se estructuran las propuestas de solución que los estudiantes discuten, defienden e intentan que se conviertan en resoluciones de la mesa, las cuales se basan en la preocupación que genera en algunos estudiantes sentimientos de angustia, lo cual se desarrolla a partir de un proceso de empatía, en el cual de una u otra manera los estudiantes se ubican en la posición del otro, de tal manera que se preguntan por el terror y el miedo que deben generar los actos terroristas que viene los pueblos ajenos a ellos.

Se considera que el elemento fundamental para poder analizar la forma en la que los estudiantes llegaron a consensos estuvo relacionado con el criterio de la claridad debido a que permitió ver un nivel argumentativo alto respecto a las propuestas para dar solución al problema, apoyados por argumentos basados en estadísticas y bien fundamentados. Ello está relacionado con la comprensión que tienen los estudiantes que participan en el modelo, de que la solución a los problemas no pasa por ser un asunto interno de cada país que debe intentar solucionar por su parte sino que se debe trabajar desde la cooperación internacional.

En relación con el grupo focal, como se ha mencionado, este se compuso de estudiantes de los tres niveles de debate y se resalta de este ejercicio la metacomprensión realizada por los estudiantes sobre su participación en el modelo. Cabe resaltar la importancia que tuvo este para verificar la relación entre algunas variables que se iba visualizando a lo largo del análisis y que se encuentran allí mencionadas. Posteriormente, se desarrolla una triangulación de los tres instrumentos, por categoría, dando como resultado que en relación con la claridad se obtienen mejores resultados respecto al nivel o grado de los estudiantes, de manera que a mayor grado de estudio se generan discursos más pertinentes o adecuados al contexto. Es importante resaltar el elemento de la claridad en la redacción de los documentos debido a que ello facilitará la claridad al transmitir el mensaje y por consiguiente facilita la discusión de posiciones y la negociación para llegar a acuerdos.

Por otra parte, se rescata la claridad como elemento fundamental del cumplimiento del propósito comunicativo de los delegados, sin embargo, es de valorar el ejercicio de balance que logran los estudiantes entre los objetivos de la delegación y del comité en el cual participan, para de esta manera demostrar la capacidad de consensos que desarrollan los estudiantes. Se debe tener en cuenta que de los elementos a resaltar en el cumplimiento del propósito se destacan las emociones de los estudiantes las cuales se pueden clasificar en dos tipos, las generadas por la participación en el debate y las generadas por la problemática tratada.

En la aplicación del modelo se realiza un ejercicio individual de empatía buscando reflexionar acerca de la forma en la que ellos vivenciarían las experiencias de los pueblos que viven los estragos de la guerra dando lugar a determinar que el modelo de naciones unidas permite avivar en el estudiante un sentimiento de 
vinculación con los individuos de la especia humana. Con base en este ejercicio surge como un interés común y es la obligación de brindar seguridad a estas poblaciones a partir del restablecimiento de sus derechos y la protección de los mismos.

En el debate se observa como la categoría de uso efectivo de la comunicación juega un rol fundamental en la manera como los estudiantes llegan a generar consensos, debido a que permite a los estudiantes tener una mejor capacidad argumentativa en el momento de negociar, defender o atacar las propuestas presentes en la mesa. Sin embargo, al mismo tiempo se observa que los estudiantes reconocen en la unión de países y el trabajo cooperativo entre los mismos, una forma para lograr su objetivo comunicativo y la solución a los problemas que abordaron en sus discusiones, de tal manera que se catalogan las instituciones supranacionales en dos grupos, a saber: en relación con el sistema de las naciones unidas o respecto a la comunidad internacional.

Sobre la competencia comunicativa se puede observar que el elemento central es la claridad en la presentación de los mensajes propuestos. Esta variable está relacionada claramente con el cumplimiento del propósito de la competencia comunicacional efectiva dirigida al consenso estudiantil.

Por otra parte, es clara la abundancia de los elementos relacionados con la categoría de ciudadanía cosmopolita, debido a la abundancia de citas relacionadas con el sistema global de derechos y su amplia relación con las instituciones supranacionales, lo que permite explicar en relación con los resultados categoriales, que los estudiantes comprenden que dicho sistema global de derechos debe ser protegido por instituciones que van más allá de las fronteras de un país.

Sin embargo, los puentes que se trazan entre estas dos super categorías están relacionados principalmente por la categoría de uso efectivo del lenguaje con la de instituciones supranacionales, en tanto que para poder generar discursos claros, que dieran respuesta a las necesidades del contexto, los estudiantes debían tener presente cuál era su contexto de dialogo, el cual era el consejo de seguridad de las naciones unidas buscando solución al problema de la guerra siria para observar la competencia comunicativa de un estudiante en el contexto adecuado. En este caso participar, el modelo de naciones unidas permite el desarrollo de la ciudadanía cosmopolita, en tanto que permite a los estudiantes adelantar un proceso investigativo que le de claridad sobre los hechos que se presentan en distintos lugares del mundo pero que afectan a la población, y es aquí que al darse cuenta de esa realidad lo lleva a despertar emociones en relación con el contexto situacional de otros seres humanos. Esta situación se valida al observar que cuando más se manifestaron emociones de los estudiantes fue en relación con un sistema global de derechos generando compromisos con el propósito de dar solución a estas situaciones problémicas.

Generalmente dichas situaciones relacionadas con los derechos son asignadas a instituciones supranacionales, y para poder dar solución se requiere entonces del consenso entre las distintas delegaciones. 


\section{Conclusiones.}

Como se ha observado, el modelo de competencias ciudadanas que ha adaptado Colombia, basado en el modelo educativo centrado en competencias, parece no ser la solución adecuada a la crisis de ciudadanía por la cual atraviesa el país. Inicialmente se debe afirmar que en la teoría se ve en la base del programa de competencias ciudadanas en Colombia, un proyecto de ciudadanía republicana, en el cual el ciudadano es participativo en la política del país. La ciudadanía práctica de Colombia no se puede entender como un tipo específico de ciudadanía, sin embargo, como se evidenció, la ciudadanía se encuentra caracterizada fundamentalmente por el predominio del interés individual sobre el bienestar colectivo- publico.

Es necesario desde la educación ayudar en la superación de dichas prácticas de ciudadanía liberal. La respuesta adecuada no se encuentra tampoco en la ciudadanía republicana y ello porque el tiempo actual en el que habitamos se encuentra permeado de manera absoluta por la dinámica globalizadora, la cual ha desarraigado a los sujetos de territorios concretos, les ha abierto la puerta a una multiculturalidad y plurinacionalidad que debe ser abordada desde el paradigma de la ciudadanía cosmopolita, en tanto que esta si bien acepta la desligación del sujeto con su territorio o nación, encuentra un nuevo punto de ligación con la humanidad como hermandad o como una comunidad que se encuentra y comparte un destino común.

El modelo de ciudadanía cosmopolita necesita de la educación como dispositivo por medio del cual se pueda preparar para la práctica de este tipo de ciudadanía. Por esta razón, se deben desarrollar maneras innovadoras de educación que habiliten a los sujetos a realizar un ejercicio adecuado de su dimensión política, es decir, del uso de sus derechos políticos y del cumplimiento de sus deberes, por ejemplo, la participación política no solo representada en el voto, sino en la discusión de los destinos planetarios del hombre.

Por lo tanto, como se ha evidenciado anteriormente, es necesario plantear un nuevo paradigma para la educación, como lo es el paradigma dialógico, que le permita al sujeto salir de las dinámicas actuales que se han planteado por los paradigmas cientificista y humanista. Aunque cabe aclarar que un nuevo paradigma traerá consigo nuevas dinámicas de enseñanza que se enfoquen en el desarrollo de la competencia comunicativa.

Es decir, la educación para la ciudadanía se debe ver desde un nuevo horizonte de sentido que puede ser el paradigma dialógico propuesto por Hoyos (2006), sin embargo, ello se debe ver reflejado en prácticas educativas concretas como lo son los modelos de naciones unidas, en los cuales los estudiantes se ven abocados al desarrollo de su competencia comunicativa para la resolución de problemas que no son empresariales o relacionados con la profesión. En este sentido, el paradigma educativo comunicacional dará cabida a dimensiones relegadas por el paradigma cientificista, como lo era la dimensión política del sujeto en tanto que esta no se encuentra mediada por objetivos comunes de empresa, sino que se encuentra mediada por intereses o necesidades comunes de la sociedad. 
Como conclusiones de la investigación se encuentra que el modelo de naciones unidas forma ciudadanía cosmopolita en la medida en que permite a los estudiantes informarse y apropiarse de una realidad distinta, es decir, el estudiante que se prepara como delegado para representar a un país, generalmente desarrolla procesos de investigación que lo conecta con procesos que ha escuchado pero que le son desconocidos en profundidad, de tal manera que se informa de otras realidades y por lo tanto toma conciencia de las mismas.

Cabe resaltar además que, el uso efectivo de la comunicación le permite al estudiante llegar a acuerdos con los otros, ello debido a que el modelo de naciones unidas genera procesos dialógicos en los cuales el estudiante pone en juego varios elementos, entre ellos sus intereses en relación con los del resto de participantes, junto con ello su emotividad la cual se expone en sus participaciones y se encuentra con las emociones de los demás participantes. Pero además de ello, se evidencia que el modelo de naciones unidas es un escenario de práctica para que los estudiantes mejoren su competencia comunicativa en tanto que se ven en la necesidad de generar discursos claros que respondan al contexto comunicativo.

Es necesario afirmar que en el modelo de naciones unidas se desarrolla ciudadanía cosmopolita, en tanto que, es un espacio que le permite al estudiante comprender a todos los seres humanos como iguales que se encuentran vinculados como familia universal y por lo tanto comprende que el aseguramiento de los derechos fundamentales de todo ser humano no dependen de un Estado. Igualmente reconoce la existencia de una serie de derechos propios de todos los seres humanos que deben ser protegidos no por los gobiernos sino por todas las personas, de tal manera que es necesaria la creación y el trabajo en comisiones internacionales que permitan el disfrute y la protección de estos derechos a todos los seres humanos, sin distinción alguna.

\section{Resultados.}

En relación con el cumplimiento de los objetivos propuestos para esta investigación se considera que se describió y analizó el modelo de las naciones unidas en relación con el desarrollo de procesos de ciudadanía cosmopolita, en tanto que durante el marco teórico se hizo un recuento y explicación de los elementos más importantes del modelo de naciones unidas, de su funcionamiento y cómo este modelo podría ser el ambiente adecuado para el desarrollo de la competencia comunicativa desde la propuesta de Guillermo Hoyos que cambia el paradigma de la educación hacia un paradigma discursivo.

Se analizó el desarrollo de la competencia comunicativa y la ciudadanía cosmopolita enfatizando en el uso efectivo del lenguaje, la comunicación de emociones, la construcción de consensos, el sistema global de derechos y las instituciones supranacionales al recolectar la información de los documentos de preparación de los estudiantes (papel de posición, discurso de apertura), la transcripción de los debates, al igual que del grupo focal se hizo el análisis categorial para evidenciar la forma en la que se desenvolvía la competencia comunicativa de 
los estudiantes participantes y la forma en la que esta permitía el desarrollo de la ciudadanía cosmopolita en los mismos. Se dieron resultados por instrumento para cada uno de esas categorías y posteriormente se realizó la triangulación de los resultados por categoría de los instrumentos.

Se construyeron y analizaron escenarios de participación donde se asumió el modelo de las naciones unidas como una estrategia pedagógica, en tanto que se diseñó y ejecutó la estrategia pedagógica del modelo de las naciones unidas para ejecutar en el año 2016. Con base en dicha ejecución se recolectó la información que permitió la creación del cuerpo textual que fue objeto de análisis durante esta investigación.

El cumplimiento de estos tres objetivos específicos permitieron que se ejecutara el objetivo general al reconocer en el modelo de las naciones unidas el desarrollo de procesos de ciudadanía cosmopolita a través de la competencia comunicativa para asumir el proyecto como una estrategia pedagógica en el Gimnasio Campestre Reino Británico. Es por esta razón que se diseñó y se ejecutó la estrategia pedagógica del modelo de las naciones unidas para el 2017, la cual tiene en cuenta varios de los elementos por mejorar que se descubrieron durante esta indagación. En otras palabras, este trabajo abre puertas a nuevos escenarios para abordar los ejes temáticos y problémicos asumidos en primer momento con esta investigación.

\section{Referencias}

Appadurai, A. (2000). Globalization. Carolina del Norte, Estados Unidos: Duke University Press.

Appiah, K. A. (2008). "Education for Global Citizenship". En: Coulter, D., Fenstermacher, G., y Wiens J.R. (eds). Yearbook of the National Society for the Study of Education. The National Society for the Study of Education, Vol. 1

Barbero, J., (1991) De los medios a las mediaciones. Barcelona: Editorial Gustavo Gili S.A.

Beck, Ulrich. (2006). Cosmopolitan visión. Cambridge: Polity.

Bennett, M., (2004). Becoming interculturally competent. En J. Wurzel (ed.), Toward multiculturalism: a reader in multicultural education (2nd ed., pp. 62-77). Newton, MA: Intercultural Resource Corporation.

Bermúdez, L., y Gonzalez, L., (2011), La competencia comunicativa: Elemento clave de las Organizaciones. Quorum Académico n. 15, v. 8, p. $95-110$.

Benéitez, J., (2010), La ciudadanía cosmopolita de Martha Nussbaum, En: Daímon. Revista Internacional de Filosofía, Suplemento 3, 2010.

Canclini, N., (1995), Consumidores y Ciudadanos: Conflictos multiculturales de la globalización, México DF: Editorial Grijalbo. 
Carvalho da Silva, M. (coord.) (2008). Pautas para una educación global. Conceptos y metodologías sobre educación global para educadores y responsables de políticas en materia educativa. España:

Centro Norte-Sur del Consejo de Europa.

Cortina, A., (1997), Ciudadanos del mundo, hacía una teoría de la ciudadanía. Madrid: Alianza editorial SA.

Delanty, G. (2006). "The cosmopolitan imagination: critical cosmopolitanism y social theory". British Journal of Sociology,57 (1), 25-47.

Delors, J. et al. (1996). Learning. the treasure within. Report to UNESCO of the International Commission on Education for the Twenty-First Century. Paris, Francia: UNESCO.

De Paz, D. (2007). Escuelas y educación para la ciudadanía global. Una mirada tranformadora. Barcelona: Intermón Oxfam Ediciones.

Escobar, G. (2007). Ética y política en el pensamiento de Adela Cortina. XIX coloquio Nacional Sobre la Enseñanza de la Filosofía. Veracruz. Recuperado el 4 de enero de 2014 de: http://galeon.com/cmpf/XIXColoquio/EscobarXIX.pdf

Gómez, H., (1999), La hipótesis del almendrón. En:¿Para dónde va Colombia? Un coloquio abierto. Bogotá: Tercer Mundo Editores.

Gutiérrez, F., (1999) Siete Proto-tesis sobre el futuro de la violencia. En Gómez Buendía, Hernando (Coord.) ¿Para dónde va Colombia? Un coloquio abierto. Bogotá: Tercer Mundo Editores.

Hymes, D., (1996) Acerca de la competencia comunicativa. Trad. Gómez Bernal Juan en Forma y función 9, pp. 13-37.

Hoyos, G., (2007), La comunicación: La competencia ciudadana. En: (Ed) Ciudadanías en formación. Bogotá: Editorial Magisterio, p. 135-172.

Jiménez, A., (2003) Democracia en tiempos de crisis 1949-1994. Bogotá: Editorial Planeta Colombiana S.A.

Kennedy, K. (1997). Citizenship Education and the Modern State. Londres: Taylor and Francis.

Lagos, T. (2003). “Global citizenship - Toward a definition”. http:// depts.washington. edu/gcp/research_pages/globalization_biblio.htm

Meyer, RM., y Sandy, LR. (2009). "Education for Global Citizenship in the New Millennium". International Journal of Diversity in Organizations, Communities, and Nations, Vol. 9, número 1, págs. 59-64 
MINISTERIO DE EDUCACIÓN NACIONAL, (2004), Estándares básicos de competencias ciudadanas: Formar para la ciudadanía. jSi es posible! Lo que necesitamos saber y saber hacer, Serie Guías No. 6. Bogotá D.C.

(2011), Orientaciones para la institucionalización de las competencias Ciudadanas. Cartilla 1. Brújula. Programa de Competencias ciudadanas. Bogotá.

Nussbaum, M., (1997), «Kant and Stoic Cosmopolitanism», Journal of Political Philosophy, no 5, 1997, pp. 1-25.

Nussbuam, M. (1997). Cultivating Humanity: A Classical Defence of Reform in Liberal Education. Cambridge, MA: Harvard University Press.

Nussbaum, M. (2006). "Education y democratic citizenship: capabilities y quality education”. Journal of Human Development, 7 (3), 385-395.

Moncada, J., (2010), Modelo Educativo Basado en Competencias. México: Trillas.

O'Byrne, D. (2003). The dimensions of global citizenship: political identity beyond the Nation-State. Londres, Reino Unido: Frank Cass.

Sen, A. (2006). Identity and Violence. The illusion of destiny. Londo: Penguin.

UNESCO. (2006). Directrices de La UNESCO sobre la educación intercultural.

UNESCO, (2014a). Global citizenship education. Preparing Learners for the challengers of the tweinty-first century. Paris, Francia: UNESCO.

Walker, M. (2006). Higher education pedagogies. Berkshire y New York: Society for Research into Higher Education y Open University Press. 